\title{
ESTIMATES FOR LOWER BOUNDS OF EIGENVALUES OF THE KLEIN-GORDON OPERATOR
}

\author{
HE-JUn SUn AND LING-ZHONG ZENG
}

\begin{abstract}
In this paper, we establish some inequalities for eigenvalues of the Klein-Gordon operator on a bounded domain in an $n$-dimensional Euclidean space. These inequalities give some sharper estimates for lower bounds of the sums of its first $k$ eigenvalues, which improve the recent results of Yildirim Yolcu (Proc. Amer. Math. Soc. 138 (2010), 4059-4066).
\end{abstract}

Mathematics subject classification (2010): 35P15.

Keywords and phrases: Eigenvalue, inequality, Klein-Gordon operator.

\section{REFERENCES}

[1] N. I. Akhiezer and I. M. Glazman, Theory of Linear Operators in Hilbert Space, Vols. I, II, Pitman, 1981.

[2] C. BANDle, Isoperimetric inequalities and applications, Pitman Monographs and Studies in Mathematics, Vol. 7, Pitman, Boston, 1980.

[3] R. Bañuelos and T. Kulczycki, The Cauchy process and the Steklov problem, J. Funct. Anal. 211 (2004), 355-423.

[4] R. BAÑUELOS AND T. KULCZYCKI, Eigenvalue gaps for the Cauchy process and a Poincaré inequality, J. Funct. Anal. 234 (2006), 199-225.

[5] F. A. BEREZIN, Covariant and contravariant symbols of operators, Izv. Akad. Nauk SSSR Ser. Mat. 37 (1972), 1134-1167.

[6] R. Blumenthal AND R. Getoon, The asymptotic distribution of the eigenvalues for a class of Markov operators, Pacific J. Math. 9 (1959), 399-408.

[7] Q.-M. CHENG, H. J. SUN, G. X. WEI AND L. Z. ZENG, Estimates for lower bounds of eigenvalues of the poly-Laplacian and quadratic polynomial operator of the Laplacian, Proc. Royal Soc. Edinburgh 143A (2013), 1147-1162.

[8] E. M. HARRELl II AND S. Yildirim Yolcu, Eigenvalue inequalities for Klein-Gordon Operators, J. Funct. Anal. 256 (2009), 3977-3995.

[9] H. KovaříK, S. Vugalter And T. Weidd, Two-dimensional Berezin-Li-Yau inequalities with a correction term, Comm. Math. Phys. 287 (2009), 959-981.

[10] A. A. ILYIN, Lower bounds for the spectrum of the Laplacian and Stokes operators, Discrete Cont. Dyn. S. 28 (2010), 131-146.

[11] A. LAPTEV AND T. WeIDL, Recent results on Lieb-Thirring inequalities, Journées "Équations aux Dérivées Partielles" (La Chapelle sur Erdre, 2000), Exp. No. XX, 14 pp., Univ. Nantes, Nantes, 2000.

[12] P. LI AND S. T. YAU, On the Schrödinger equations and the eigenvalue problem, Comm. Math. Phys. 88 (1983), 309-318.

[13] E. LIEB, The number of bound states of one-body Schröinger operators and the Weyl problem, Proc. Symp. Pure Math. 36 (1980), 241-252.

[14] A. D. Melas, A lower bound for sums of eigenvalues of the Laplacian, Proc. Amer. Math. Soc. 131 (2003), 631-636.

[15] G. PÓlya, On the eigenvalues of vibrating membranes, Proc. Lond. Math. Soc. 11 (1961), 419-433.

[16] G. PÓLYA AND G. SZEGÖ, Isoperimetric inequalities in mathematical physics, Annals of mathematics studies, No. 27, Princeton University Press, Princeton, 1951. 
[17] G. TALENTI, Inequalities in rearrangement-invariant function spaces, in: Nonlinear Analysis, Function Spaces and Applications, Vol. 5, pp. 177-230, Prague, Prometheus, 1995.

[18] T. WEIDL, Improved Berezin-Li-Yau inequalities with a remainder term, Spectral Theory of Differential Operators, Amer. Math. Soc. Transl. Ser. 2, 225 (2008), 253-263.

[19] H. WEYL, Das asymptotische Verteilungsgesetz der Eigenwerte linearer partieller Differentialgleichungen (mit einer Anwendung auf die Theorie der Hohlraumstrahlung), Math. Ann. 71 (1912), 441479.

[20] S. Yildirim Yolcu, An improvement to a Brezin-Li-Yau type inequality, Proc. Amer. Math. Soc. 138 (2010), 4059-4066.

[21] T. YoLCU, Refined bounds for the eigenvalues of the Klein-Gordon operator, To appear in Proc. Amer. Math. Soc. 